\title{
The enigmatic outburst of V445 Puppis - A possible helium nova?
}

\author{
N. M. Ashok and D. P. K. Banerjee
}

\author{
Physical Research Laboratory, Navrangpura, Ahmedabad 380 009, India \\ e-mail: orion@prl.ernet.in
}

Received 22 January 2003 / Accepted 16 July 2003

\begin{abstract}
J H K$ spectroscopic and photometric observations are reported for the enigmatic, nova-like, variable V445 Puppis which erupted at the end of 2000. The near-IR spectra are hydrogen-deficient and unusually rich in CI lines. The important CI lines are found to be positioned at 1.133, 1.166, 1.175, 1.189, 1.26 and $1.689 \mu \mathrm{m}$. Model calculations for the CI lines are done to make the identification of the lines secure. Photometric data, taken on January 2.90 UT, 2001 shortly after the outburst, show the formation of an optically thin dust shell around V445 Puppis. The temperature and upper limit for the mass of the dust shell are estimated to be $1800 \mathrm{~K}$ and $M_{\text {dust }} \sim 10^{-9} M_{\odot}$ respectively. A subsequent episode of massive dust formation indicated by the observed, deep visual dimming - is also seen in JHK images of early November 2001. V445 Puppis has shown two unusual properties for a nova: (i) the hydrogen-deficiency and $\mathrm{He} / \mathrm{C}$ enrichment of the object as seen from optical and IR data and (ii) synchrotron radio emission which was detected nearly a year after its outburst by other workers. The strange nature of the object is discussed and it is shown that it is difficult to place it in the known categories of eruptive variables viz. novae (classical, recurrent or symbiotic); born-again AGB stars; the new class of eruptive variables like V838 Mon; and $\mathrm{RCB} / \mathrm{HdC}$ stars. Tentative evidence for an accretion disk and binarity of the V445 Puppis system is presented. It is debated whether V445 Puppis is a rare helium nova or a unique object.
\end{abstract}

Key words. stars: individual: V445 Puppis - infrared: stars - stars: novae, cataclysmic variables - techniques: spectroscopic

\section{Introduction}

V445 Puppis - a nova like object - was first reported to be in outburst on 30 December 2000 by Kanatsu (Kato \& Kanatsu 2000). The exact time of the object's eruption is not certain an aspect that is discussed in Sect. 3.1. Spectra in the visible region, taken in the early stages after the detection, showed many permitted lines of FeII, CaI, CaII, OI and NaI (Fujii 2001; Liller 2001a,b; Shemmer et al. 2001; Wagner et al. 2001a,b). A striking feature of the optical spectra was the absence of hydrogen lines in the spectra. Near-IR spectra reported here also confirm the absence of Paschen and Brackett hydrogen lines in the $J H K$ bands. The deficiency of hydrogen in V445 Puppis shows that it is not a usual nova and shows the strangeness of the object. This point is also made by Wagner et al. (2001a) who state that the optical spectrum of V445 Puppis is not typical of classical FeII type novae, recurrent novae or symbiotic novae. Mid-IR $(3-14 \mu \mathrm{m})$ spectroscopy by Lynch et al. (2001) reveal only a smooth featureless continuum deviating significantly from a black-body distribution and which can be explained by invoking the presence of dust. Another interesting development in the evolution of V445 Puppis was the detection at radio wavelengths almost a year after the outburst (Rupen et al. 2001a). Radio emission has been seen in a few

Send offprint requests to: N. M. Ashok, e-mail: ashok@prl.ernet.in novae (Seaquist 1989) but such emission has the characteristics of thermal bremsstrahlung radiation emitted by ionized gas. In V445 Puppis, non-thermal synchrotron radiation is seen which is rather rare (another example of this being the old nova remnant GK Persei). Thus V445 Puppis has all the hallmarks of being a very interesting object.

In this work, we mainly present spectroscopic results (and also some photometric data) from $J H K$ observations of V445 Puppis made at five fairly evenly spaced epochs. These should help in following the temporal evolution of V445 Puppis and understanding its puzzling nature. From our results it would appear that V445 Puppis is a unique object and could be a helium nova. A helium nova is believed to occur due to a thermo-nuclear runaway on the surface of a degenerate white dwarf accreting helium from its helium-rich companion (Kato et al. 1989). The nova ejecta, in such an outburst, are expected to be hydrogen-deficient as found in V445 Puppis.

\section{Observations}

Near-IR JHK spectra at a resolution of $\sim 1000$ were obtained at the Mt. Abu $1.2 \mathrm{~m}$ telescope using a Near Infrared Imager/Spectrometer with a $256 \times 256 \mathrm{HgCdTe}$ NICMOS3 array. We present here the spectroscopic observations of five days viz. January 1.63 UT, January 20.81 UT, February 1.73 UT, February 18.70 UT and March 1.66 UT, 2001. In each of the $J$, $H$ and $K$-bands a set of at least two spectra were taken. In each 


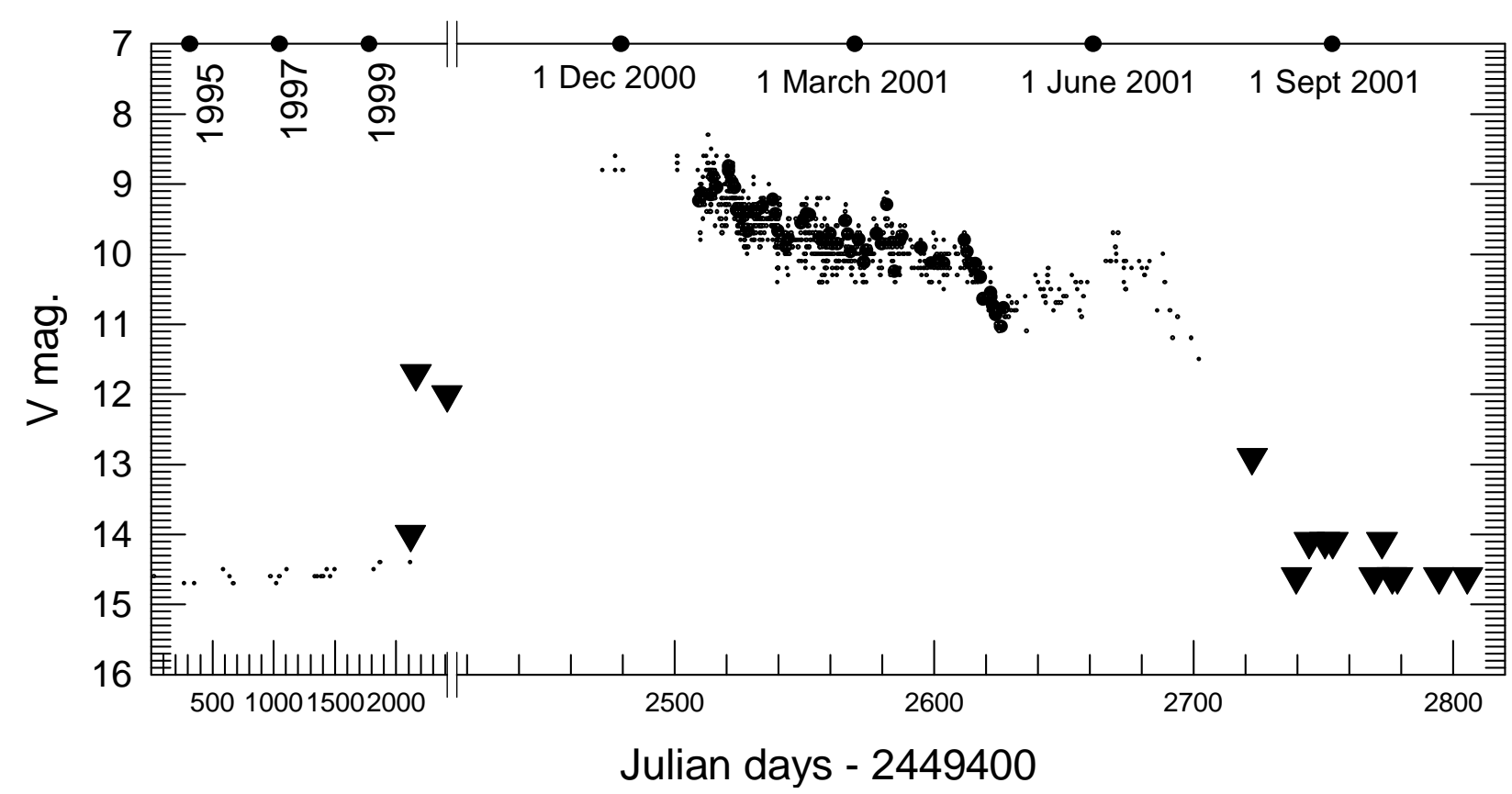

Fig. 1. The $V$-band lightcurve of V445 Puppis between 1994 to 2001 based on IAU circulars and VSNET reports. The small dots represent visual/photographic estimates and the circles represent photo-electric/CCD values. The triangles give upper limits on the magnitudes.

set the star was offset to two different positions of the slit (slit width $\left.=2^{\prime \prime}\right)$. The signal-to-noise ratio of the spectra, as determined using IRAF, is moderate and ranges between 30 to 60 in the $J H K$-bands. The exposure times for the spectra were typically between 60 to $120 \mathrm{~s}$. Spectral calibration was done using the $\mathrm{OH}$ sky lines that register with the spectra. The comparison star HR 2988 was used for dividing the spectra in all cases. We believe the telluric lines, present in the spectra, have been well removed in the process of division. This is because of (i) the close proximity of HR 2988 to V445 Puppis and (ii) also the small time gap (typically 10-15 $\mathrm{min}$ ) between observing the spectra of V445 Puppis and HR 2988 in any of the JHKbands. This ensures thatboth the stars were at a similar airmass. The dividing process should thereforeremove the telluric lines reasonably well.

Photometry in the $J H K$-bands was performed on January 2.90 UT and November 1.96 UT, 2001 using the NICMOS3 array, mentioned above, in the imaging mode. The sky was photometric on both days with a typical seeing of $1^{\prime \prime}$. Several frames in 4 dithered positions, offset from each other by $30^{\prime \prime}$, were obtained in all the filters. The times for all the individual frames was $100 \mathrm{~ms}$ on 2 Jan. 01 and ranged between 30 to $60 \mathrm{~s}$ on 1 Nov. 01. The total integration times in each of the dithered positions are as follows (i) $400 \mathrm{~ms}$ in each of the $J H K$-bands for 2 Jan. 01 and (ii) $3 \mathrm{~min}$ in $J$ and $90 \mathrm{~s}$ in $H$ on 1 Nov. 01. The sky frames were generated using these dithered frames. The mean air-mass at the time of observations for V445 Puppis was 1.79 on 2 Jan. 01 and 1.59 on 1 Nov. 01. The UKIRT standard star HD 77281 was used for photometric calibration on 2 Jan. 01 and observed soon after V445 Puppis. As a crosscheck, HR 2956 ( $V=6.50$, spectral type B7V) was also observed. The adopted $J H K$ magnitudes for HR 2956 were taken from the 2MASS survey to be $J=6.642, H=6.723$ and
$K=6.686$. For the 1 Nov. 01 observations, FS $13(J=10.517$, $H=10.189$ and $K=10.137$ ) was used as the standard star (Hunt et al. 1998). The atmospheric extinction corrections were done assuming average values of $k_{J}=0.15, k_{H}=0.15$ and $k_{K}=0.1 \mathrm{mag}$ for the Mt. Abu Observatory site. The results from the photometry are presented below (Table 2). The nearIR photometric and spectroscopic data were all reduced using IRAF.

\section{Results}

\subsection{The light curve of V445 Puppis}

The outburst date of V445 Puppis is uncertain but can be constrained from the VSNET reports. This part of the sky has been monitored by Takamizawa between March 1994 and December 1999 and he does not find any object brighter than 14 mag on his photographic plates (IAUC 7552). Subsequent photographic records from VSNET (http://www.kusastro.kyoto-u.ac.jp.vsnet) show that the object was fainter than 12th mag on 26 September 2000 and had brightened to 8.8 mag by 23 November 2000 . Thus the outburst occurred between 26 Sept. and 23 Nov. 2000. Lynch et al. (2001) have concluded, possibly by mistake, that the outburst occurred between 3 and 31 December 2000. The progenitor of V445 Puppis has been identified by Platais et al. (2001) as a 13.6 mag star (identification number 22727221) in the US Naval Observatory CCD Astrograph Catalog (UCAC1). In the USNO A2.0 catalog, the corresponding identification number is 0600-06937901 with the blue and red magnitudes listed as 13.8 and 13.2 respectively. Figure 1 shows the evolution of the light curve for V445 Puppis. While the decline of the light curve is similar to that of a slow nova, the outburst 


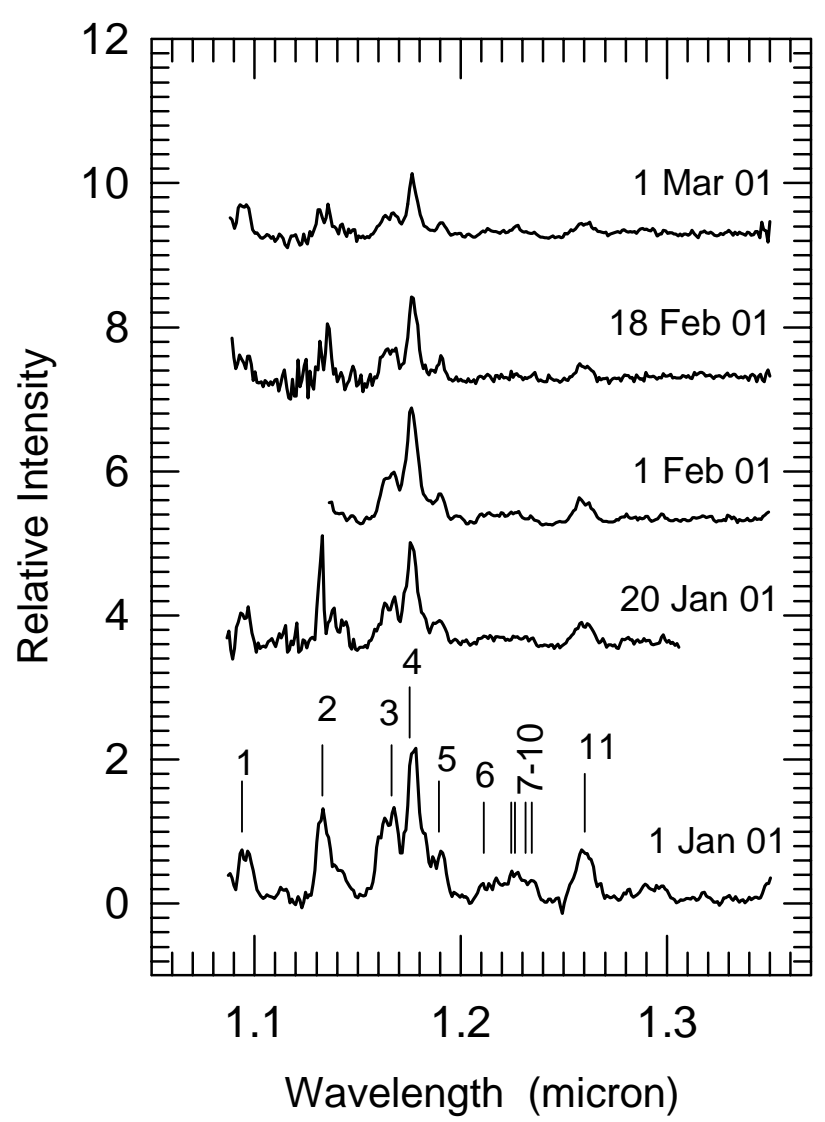

Fig. 2. The $J$-band spectra of V445 Puppis are shown at different epochs. The spectra have been offset from each other for clarity. The identified lines are marked and numbered - their details are given in Table 1.

amplitude of approximately six magnitudes is small for a classical nova. Other differences between the object and classical novae are discussed in Sect. 4.

\subsection{JHK spectroscopy}

The main thrust of this work has been in the $J H K$ spectroscopy of V445 Puppis. The object was observed at regular intervals between 1 Jan. to 1 March 2001. The JHK spectra are shown in Figs. 2-4. Of these, the more interesting spectra are in the $J$-band where several emission lines can be seen. The emission lines that are seen in the $J$ and $H$ spectra are numbered in Figs. 2 and 3. Their identification and other relevant details, are listed in Table 1. Almost all the lines that are present appear to be due to neutral carbon. The identification of the CI lines is done on two bases. First we have compared the strengths and positions of these lines with the laboratory IR spectra of CI as given by Johansson \& Litzen (1965) and Johansson (1966) and a good match is found. Furthermore, we have computed a simple model spectrum that should arise from CI emission from the shell. For this spectrum, we have selected 170 of the strongest CI lines between 1.08 and $2.5 \mu \mathrm{m}$ with their transition probabilities, from the atomic line list compiled by Kurucz (http: //kurucz . harvard. edu/linelists.html). The strength of any line is computed as follows. If the line

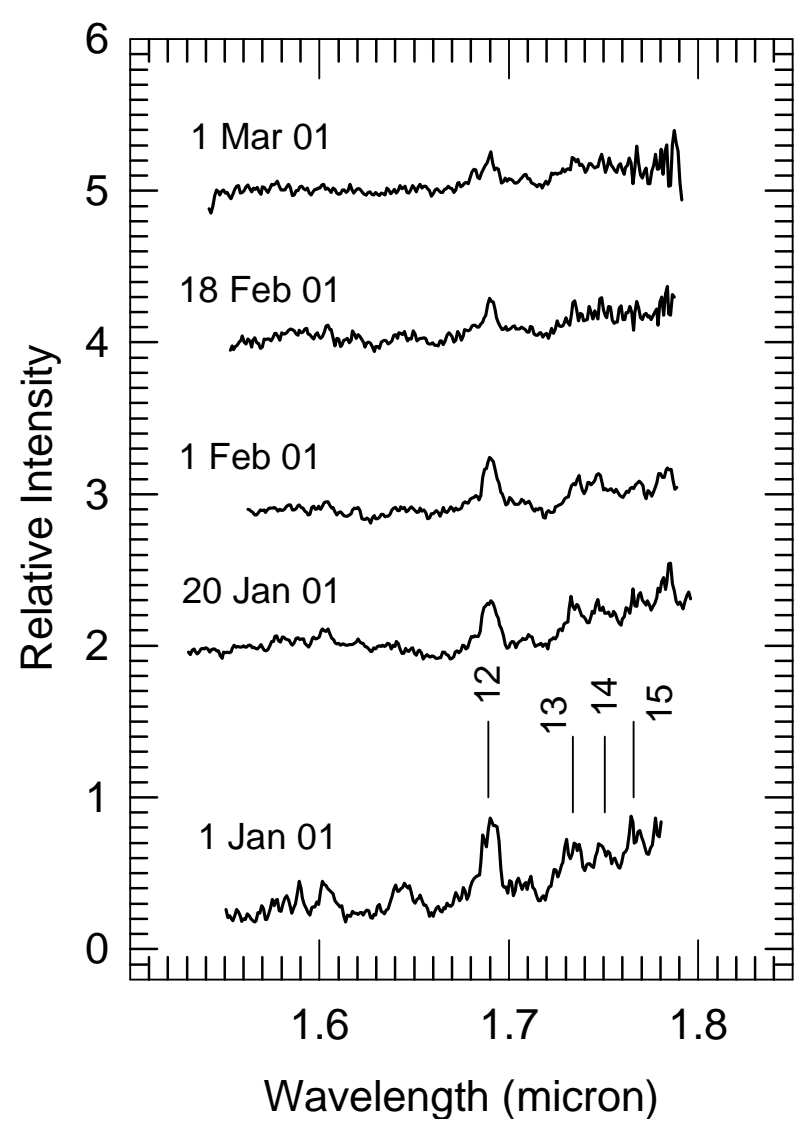

Fig. 3. The $H$-band spectra of V445 Puppis are shown at different epochs. The spectra have been offset from each other for clarity. The identified lines are numbered and marked - their details are given in Table 1.

originates in a downward transition from level 2 to 1 then, the luminosity of the line $L_{\text {line }}$ is given by:

$L_{\text {line }}=N_{2} A_{21} h v V$

where $N_{2}$ is the number density of CI atoms in the excited state 2, $A_{21}$ is the Einstein coefficient of spontaneous emission, $h v$ is the energy of the emitted photon and $V$ is the volume of the emitting gas.

From Eq. (1), it is seen that, the strength of any line will essentially depend only on $A_{21}$ and $N_{2}$. If the populations of the upper level $\left(N_{2}\right)$ for the different CI lines are temporarily assumed to be the same, then the line strength depends basically only on $A_{21}$. Knowing the transition probabilities, we have computed the relative line strengths from Eq. (1). We assume that the shape of each line can be reasonably represented by a Gaussian whose FWHM has been chosen to be approximately $1500 \mathrm{~km} \mathrm{~s}^{-1}$ - representative of the observed linewidths in V445 Puppis. Co-addition of all the Gaussians - corresponding to all the lines - yields a model spectrum which is shown in Fig. 5 (labeled as model 1). The observed $J$-band spectrum of $1 \mathrm{Jan} .01$, in which the emission lines are most prominent, is also shown in the same figure for comparison. A more accurate calculation has taken into account the population of the upper levels $N_{2}$ in calculating the relative line strengths. Assuming 


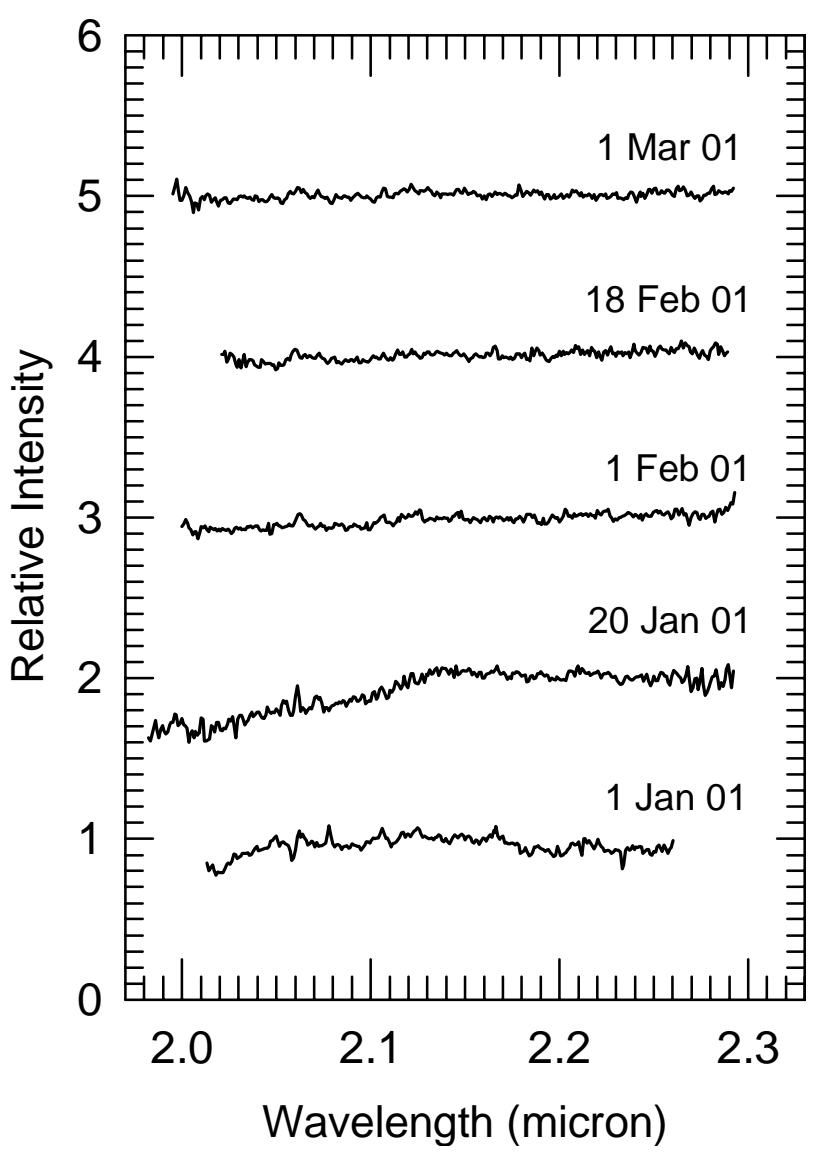

Fig. 4. The $K$-band spectra of V445 Puppis are shown at different epochs. The spectra have been offset from each other for clarity.

the emitting gas to be in local thermal equilibrium, $N_{2}$ will be given by a Boltzmann distribution viz.

$N_{2} / N(C)=\left(g_{2} / U\right) \mathrm{e}^{\left(-\chi_{02} / \mathrm{kT}\right)}$

where $U$ is the partition function, $T$ is the temperature of the gas, $\chi_{02}$ is the energy difference between the ground state and level $2, g_{2}$ is the statistical weight of the upper level and $N(C)$ is the total number density of the carbon atoms. The energy level values for the different states (to compute $\chi_{02}$ ) and $g_{2}$ values have been taken from Kurucz's line list. The partition function values, for different temperatures, have been taken from Allen (1976) and Aller (1963).

Nova shell ejecta have temperatures typically in the range 4000-5000 K (Williams 1994). However we find that slightly higher temperatures reproduce the observed spectra better. In particular, the model spectrum computed for $T=8000 \mathrm{~K}$ (labeled as model 2) isalso shown in Fig. 5. Changes in the adopted temperature do change the relative strengthof the different lines, but marginally, and not in any drastic fashion. However, the primary purpose of the simulated spectra of Fig. 5 is only to secure the identification of the CI lines. Figure 5 only shows graphically the expected positions of the CI lines and gives a rough simulation of their expected strengths. As may be seen, all the strong CI lines are reproduced, and to a large extent even the weaker lines. Based on the results of Fig. 5, it would appear reasonable to identify most of the observed lines

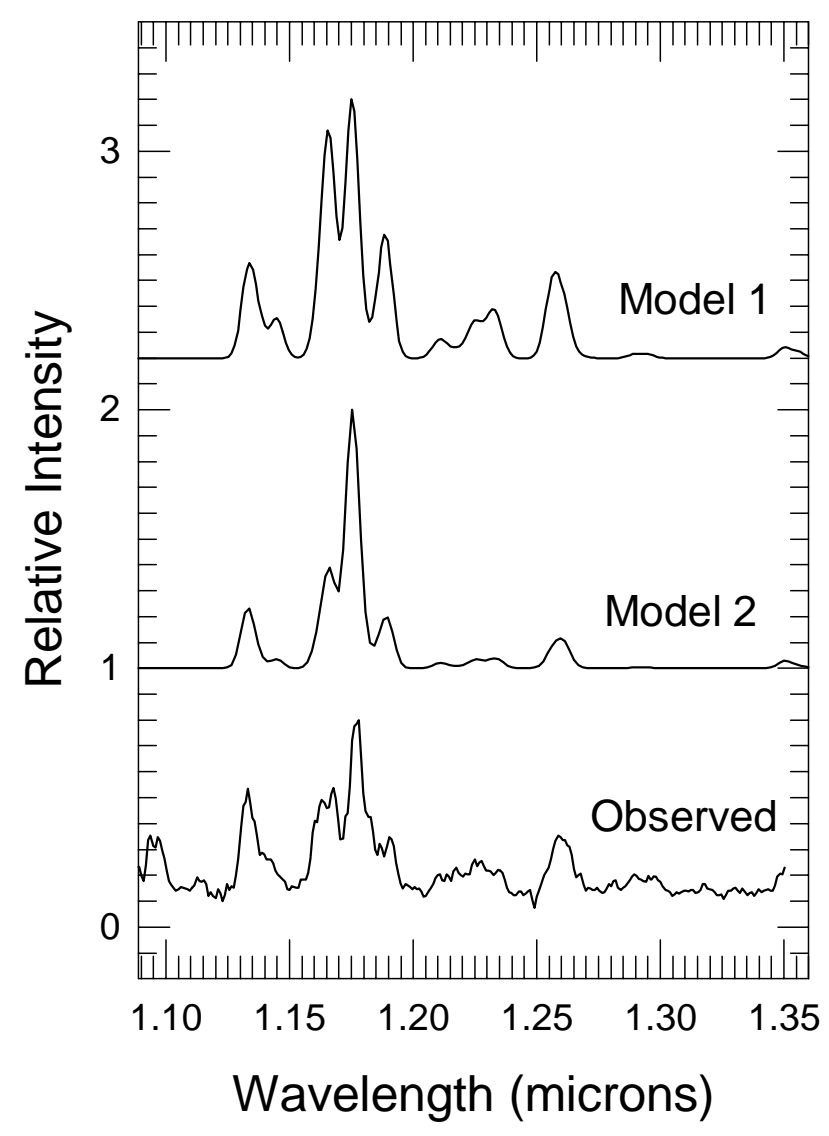

Fig. 5. Model spectra compared with the $J$-band spectra of 1 Jan. 01. Thedetails are available in the text of Sect. 3.2.

in the $J$-band with CI. The model spectra for $H$ and $K$ have also been computed but are not shown here. The model results for the $H$-band show the strongest CI line to be at $1.69 \mu \mathrm{m}$ which matches what is observed at that wavelength. A few other lines between 1.7 to $1.8 \mu \mathrm{m}$ are predicted as well (weaker than the $1.69 \mu \mathrm{m}$ line). Their positions have been marked in the $H$-band spectra of Fig. 3 and these lines are also seen in our data. In the $K$-band no line of any significant strength is predicted. This agrees with the rather featureless $K$-band spectra that we observe (Fig. 4).

In Table 1, we have given the equivalent widths for the emission features seen in the spectra of 1 Jan. 01 where the lines are the strongest. Since some of the features are blended, we have given the combined equivalent width of the blend. In particular, combined equivalent widths have been given in Table 1 for features 3 to 5 and 6 to 10 which are blended. The reality of the features can be judged by comparing their equivalent widths with those of modulations in the continuum due to noise. The equivalent width values for such modulations are in the range of 3 to $7 \AA$ in the $J$ and $H$-band spectra. In comparison, on 1 March 2001, when the lines were the weakest, the equivalent widths for the features which are clearly discernible viz. $1,2,3-5,6-10,11$ and 12 are 25, 39, 102, 27, 22 and $41 \AA$ respectively. The modulations in the continuum have similar equivalent widths as on 1 Jan. 01 . In general, the $H$-band features are a little noisy except for the $1.689 \mu \mathrm{m}$ line. 
Table 1. A list of the lines identified from the $J$ and $H$ spectra shown in Figs. 2 and 3. Please refer to the text for greater details on the equivalent widths listed below for 1 January 2001. (u.i = unidentified).

\begin{tabular}{|c|c|c|c|c|}
\hline $\begin{array}{l}\text { Line \# } \\
\text { (from Figs. } 2 \text { and 3) }\end{array}$ & $\bar{~} \overline{\lambda(\mu \mathrm{m})}$ & 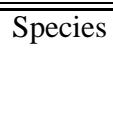 & $\begin{array}{l}\text { Other } \\
\text { contributors }\end{array}$ & $\begin{array}{l}\text { Eq. } \\
\text { width } \\
(\AA)\end{array}$ \\
\hline 1 & 1.0938 & CII & & 34 \\
\hline 2 & 1.1330 & CI & CII & 110 \\
\hline 3 & $\begin{array}{l}1.1658 \\
1.1669\end{array}$ & $\begin{array}{l}\text { CI } \\
,\end{array}$ & CII & 320 \\
\hline 4 & $\begin{array}{l}1.1748, \\
1.1753, \\
1.1755\end{array}$ & $\begin{array}{l}\text { CI } \\
" \\
״\end{array}$ & & \\
\hline 5 & 1.1895 & CI & & \\
\hline 6 & 1.2112 & CI & & 69 \\
\hline 7 & $\begin{array}{l}1.2244 \\
1.2248\end{array}$ & $\begin{array}{l}\text { CI } \\
,\end{array}$ & & \\
\hline 8 & 1.2264 & CI & & \\
\hline 9 & 1.2314 & CI & & \\
\hline 10 & $\begin{array}{l}1.2336 \\
1.2348\end{array}$ & $\begin{array}{l}\text { CI } \\
"\end{array}$ & & \\
\hline 11 & $\begin{array}{l}1.2581, \\
1.2601, \\
1.2614\end{array}$ & $\begin{array}{l}\text { CI } \\
, \\
״\end{array}$ & & 65 \\
\hline 12 & 1.6890 & CI & CII & 53 \\
\hline 13 & 1.7338 & CI & & 15 \\
\hline 14 & 1.7505 & CI & & 9 \\
\hline 15 & 1.7659 & u.i & & 12 \\
\hline
\end{tabular}

While it appears that $\mathrm{CI}$ is the main contributor to the $J H K$ spectra, it is possible that there is some contribution from CII also. This is specially so because CII lines have been seen in the optical spectra, as will be discussed shortly. We have determined some of the stronger CII lines (based primarily on their transition probabilities) in the $J H K$ region from Kurucz's line list. It will need a detailed model - beyond the scope of this work - to calculate the absolute strengths of these CII lines vis-a-vis the CI lines. But their expected wavelength positions should give some indication whether they are present in the JHK spectra or not. The expected CII lines are found to lie at wavelengths of 1.093, $1.144,1.158,1.674,1.700$ and $2.222 \mu \mathrm{m}$. There is a line at $1.09 \mu \mathrm{m}$ which is consistently seen in theobserved spectra this could be due to CII. Of the other CII lines the 1.144, 1.158 and $1.700 \mu \mathrm{m}$ lines may be present in theobserved spectra. The 1.144 and $1.158 \mu \mathrm{m}$ CII lines could be lying onthe wings of the observed 1.133 and $1.165 \mu \mathrm{m}$ CI lines - giving these a slightly broader appearance. Similarly, the expected $1.700 \mu \mathrm{m}$ CII line may be blended with the $1.689 \mu \mathrm{m}$ CI line. Thus there is someevidence for the presence of CII in the $J H K$ spectra.

It must be mentioned that in an earlier IAU circular (Ashok \& Banerjee 2001) we had attributed some of the CI lines reported here to CIII and CIV. This was because some CIII and CIV lines do occur at similar wavelengths as CI - e.g. as seen in the spectra of Wolf-Rayet stars (Eenens et al. 1991). But some of these strong CIII and CIV lines are not seen here. Furthermore, since lines from low-ionization species have been definitely identified in the optical spectra of V445 Puppis, it is more consistent to attribute the observed emission lines to CI (with some contribution from CII possibly), rather than highionization species like CIII or CIV.

It may be seen from the $J H K$ spectra that V445 Pup is hydrogen-deficient. In the $K$-band there is no sign of the Brackett gamma $(\mathrm{Br} \gamma)$ line at $2.1656 \mu \mathrm{m}$. Similarly other Brackett series lines (Brackett 10 to 19) which are commonly seen in the $H$-band in the spectra of classical novae are missing. Paschen beta $(\mathrm{Pa} \beta)$ at $1.2818 \mu \mathrm{m}$ is also not present in the $J$-band spectrum. The only possible indication for the presence of hydrogen is the $1.09 \mu \mathrm{m}$ line which coincides with Paschen gamma (Pa $\gamma$ ). However, $\mathrm{Pa} \gamma$ is expected to be weaker than $\mathrm{Pa} \beta$ which, in the present data, is completely absent. Hence it is difficult to attribute this line to hydrogen $\mathrm{Pa} \gamma$ and it is more likely to be due to $\mathrm{CII}$ as discussed earlier.

We do not find the signature of helium lines in the $J H K$ spectra. However, the presence of $\mathrm{He}$ is more conclusively seen in the optical spectra wherein several HeI lines are prominently seen (Wagner et al. 2001c) while at the same time the hydrogen deficiency is manifested by the weakness/absence of the Balmer hydrogen lines. It is also important to note that many carbon lines are also strongly seen in the optical spectra (Kamath \& Anupama 2002; Wagner's site at http://vela.as.arizona.edu/ rmw/v445pup.html) at different epochs after the outburst. In fact in a sample spectrum by M. Fujii, Bisei Observatory, taken soon after the outburst, the strongest optical line that is seen is the CII $6582 \AA$ line (http://www1.harenet.ne.jp/aikow/0113pnp.gif).

The Fujii spectrum also shows the presence of the HeI lines. Thus it would be fair to qualitatively say that the IR and optical evidence indicate that not only is V445 Puppis a hydrogen-deficient object but also that it is rich in carbon and helium.

\subsection{Distance and reddening}

We briefly discuss the distance and reddening towards V445 Pup since these parameters enter the photometric calculations described in the next subsection. The distance to V445 Pup is poorly known. The only estimate for the distance $d$ is by Wagner (2001) who gives an upper limit of $3 \mathrm{kpc}$ based on the strength of an interstellar absorption line at $5780 \AA$ (http://vela.as. arizona. edu/ rmw/v445pup.html).

For such an adopted upper limit for $d$, the extinction maps by Neckel et al. (1980) give a value of $E(B-V) \sim 0.25$ in the direction of V445 Puppis. This value is derived by converting Neckel et al.'s (1980) values of $A_{V}$ using the relation $A_{V}=3.1 E(B-V)$ (Koornneef 1983). As an additional confirmation for $E(B-V)$, we also used $U B V$ photometric data for 110 stars by Wooden (1970) in an approximately $5 \times 5$ degree field around V445 Puppis. Since the spectral type is known, and hence the intrinsic $(B-V)_{0}$ color, the excess color $E(B-V)$ can be found from the observed $(B-V)$ color. $A_{V}$ can hence be found. The distance can then be determined by the standard relation $m_{V}-M_{V}=5 \log d-5+A_{V}$. The values for the intrinsic $(B-V)_{0}$ colors and absolute magnitudes, for different 


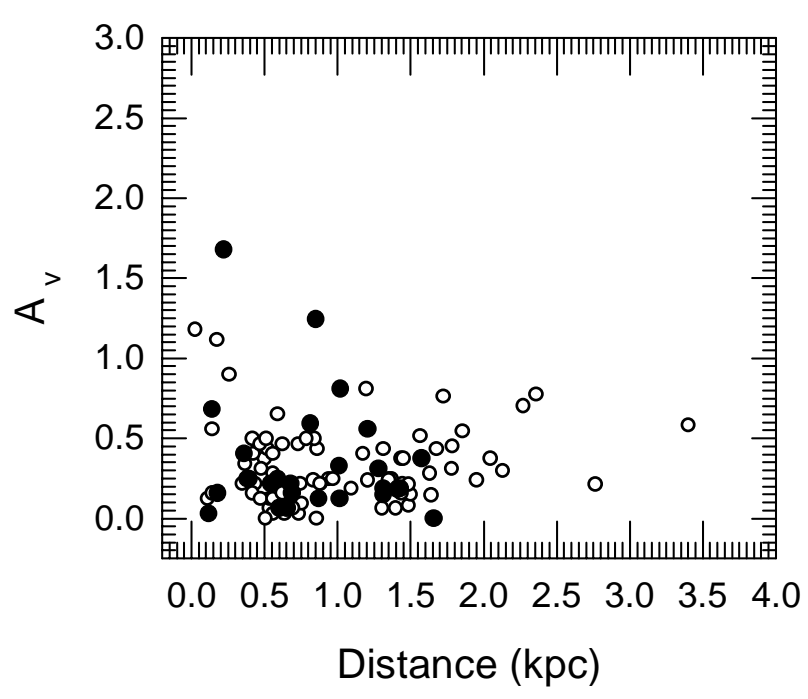

Fig. 6. An $A_{V}$ versus distance plot for stars in a $5 \times 5$ degree field around V445 Puppis based on data from Wooden (1970). The stars within a smaller, 1 degree field of V445 Puppis are plotted by filled circles, the other stars by empty circles.

Table 2. JHK photometry of V445 Puppis.

\begin{tabular}{llll}
\hline \hline Obs. date (UT) & $J$ & $H$ & $K$ \\
\hline 2.90 Jan. 2001 & $6.35 \pm 0.06$ & $5.20 \pm 0.03$ & $4.41 \pm 0.12$ \\
1.96 Nov. 2001 & $>15.5$ & $12.6 \pm 0.20$ & - \\
\hline
\end{tabular}

spectral types, were taken from Lang (1991) and Allen (1976). We have plotted in Fig. 6 the $A_{V}$ versus distance relation based on the Wooden (1970) data. Since a $5 \times 5$ degree field is rather large and extinction can vary considerably along any line of sight in it, we have also shown in Fig. 6 the extinction for stars within a smaller, 1 degree field of V445 Puppis. Based on the results of Fig. 6 and also on the Neckel et al. (1980) data, we feel it reasonable to adopt a value of $E(B-V)=0.25$ for V445 Puppis, unless the distance to it is much greater than $3 \mathrm{kpc}$. It may be noted, that the extinction in this direction is generally low.

\subsection{Near Infrared photometry}

The details and results of the photometry are given in Table 2. The $J H K$ magnitudes listed are the observed magnitudes. We have used Koornneef's (1983) relations viz. $A_{V}=3.1 E(B-V)$, $A_{J}=0.265 A_{V}, A_{H}=0.155 A_{V}$ and $A_{K}=0.090 A_{V}$ to correct for interstellar extinction. Absolute flux calibration was done by adopting zero magnitude fluxes from Koornneef (1983). We have plotted the spectral energy distribution (SED) of V445 Puppis for 2 Jan. 01, aftercorrecting for $E(B-V)=0.25$, in the upper panel of Fig. 7. In this figure the $J H K$ fluxes are from the present work whereas the $U B V R$ values are taken from Gilmore (2001). It may be noted that Gilmore's (2001) observations are for Jan. 6.58 UT, 2001 and slightly separated in time from our $2 \mathrm{Jan} .01 \mathrm{JHK}$ observations. However, since both sets of observations are almost simultaneous, no significant error is expected to be introduced in the results of Fig. 7. This is especially so, since as seen from the light curve

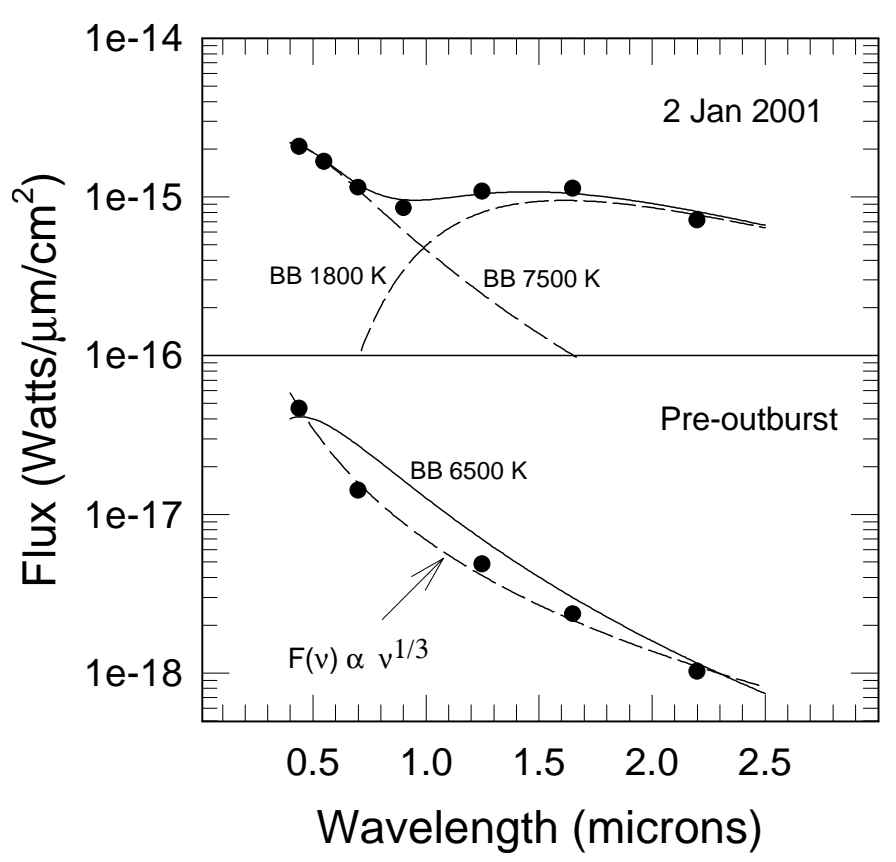

Fig. 7. The post-outburst energy distribution of V445 Puppis on 2 Jan. 01 is shown in the top panel. It has been matched by the sum of 2 black-body curves - one at $7500 \mathrm{~K}$ and the other for a dust component at $1800 \mathrm{~K}$. The bottom panel shows the pre-outburst flux distribution based on 2MASS data. A $6500 \mathrm{~K}$ black-body fit is shown to this and also the expected distribution from an accretion disk following a $F_{v} \alpha v^{1 / 3}$ relation. For greater details see text.

of Fig. 1, V445 Puppis was not showing strong photometric changes around this time.

The SED of 2 Jan. 01 could not be fit by a single blackbody curve as it shows a significant IR excess. Accordingly it has been fit by a $7500 \mathrm{~K}$ black-body component (corresponding to V455 Puppis proper) and a cooler component of temperature $1800 \mathrm{~K}$. The cooler component arises from dust that has formed around V445 Puppis. The presence of dust could give an additional extinction apart from the interstellar extinction corresponding to the adopted value of $E(B-V)=0.25$. In view of this we have calculated various combinations of the SED that result for variations of $E(B-V)$ from 0.25 to 0.5 ; the hot blackbody component from 6000 to $12000 \mathrm{~K}$ and the cool blackbody component (dust) from 1200-2200 K. We find that $E(B-V) \sim 0.25$ still continues to give an optimal fit to the data, indicating that there is no significant extinction from the dust. We also find that almost equally good fits to the data are found for a hot component temperature in the range $7500-8500 \mathrm{~K}$ combined with dust temperature in the range $1750-1850 \mathrm{~K}$. We believe a dust temperature of $1800 \mathrm{~K}$ is fairly representative of the observed data and adopt this value for subsequent calculations for the mass of the dust shell.

Lynch et al. (2001) have also found evidence for the presence of dust from their 3-14 $\mu \mathrm{m} L M N$ band spectroscopy of 31 January 2001. However, they conclude that the dust most likely existed before the outburst ratherthan having been created post-outburst. The premise for this inference is that dust could not have formed so early after the outburst. But, as mentioned earlier in Sect. 3.1, Lynch et al. (2001) have placed the 
outburst date a month later than what we believe to be correct. In classical novae, the time scale for dust to form is typically 50-70 days after outburst (Gehrz 1988). However there are cases like V838 Her (Chandrasekhar et al. 1992 and references therein) when dust formed as early as eight days after outburst. In the case of V445 Puppis, we feel that sufficient time after the outburst may have elapsed for dust to form and that the dust that is seen here has been created post-outburst. This view is bolstered by looking at the pre-outburst SED of V445 Puppis shown in the lower panel of Fig. 7. Here, the preoutburst fluxes are given by the filled circles which are derived from the $B$ and $R$ magnitudes (from the USNO A2.0 database) and the $J H K$ magnitudes from the 2MASS survey. As may be noted there is no sign of a pronounced IR excess in the preoutburst SED of V445 Puppis. Discussion of the other plots in the lower panel of Fig. 7 is deferred to Sect. 4.

The mass of the dust shell can be inferred from the infrared excess that is seen in the data of 2 Jan. 01. Following Woodward et al. (1993), the mass of the dust shell (in units of $M_{\odot}$ ) is given by

$M_{\text {dust }}=1.1 \times 10^{6}\left(\lambda F_{\lambda}\right)_{\max } d^{2} / T_{\text {dust }}^{6}$.

In the above equation, $T_{\text {dust }}$ is the black-body temperature of the dust in units of $10^{3} \mathrm{~K}, d$ is the distance to the object in kpc and $\left(\lambda F_{\lambda}\right)_{\max }$ is the flux, in $\mathrm{W} \mathrm{cm}^{-2}$, measured at the peak of the SED for the dust. Equation (3) assumes the dust is composed of carbon particles of size $\leq 1 \mu \mathrm{m}$ and having a density $2.25 \mathrm{gm} / \mathrm{cm}^{3}$. The assumption that the dust is made of carbon particles may be quite valid in the case of V445 Puppis because of the strong presence of carbon in the optical/IR spectra. Further, the mid-IR spectra of Lynch et al. (2001) show a featureless continuum which is generally attributable to dust in the form of carbon/graphite. From the data for 2 Jan. 01 we get $M_{\text {dust }}=1.8 \times 10^{-10} d^{2}$ for $T_{\text {dust }}=1800 \mathrm{~K}$ and $\left(\lambda F_{\lambda}\right)_{\max }=$ $1.716 \times 10^{-15} \mathrm{~W} \mathrm{~cm}^{-2}$. For a maximum value of $d=3 \mathrm{kpc}$, the upper limit of the mass of the dust shell is found to be $M_{\text {dust }}=1.62 \times 10^{-9} M_{\odot}$.

Regarding the photometric data for 1 Nov. 01, an accurate estimate could not be made of the $K$-band magnitude because of inadequate $\mathrm{S} / \mathrm{N}$ in the images. However the object is clearly seen in the $K$-band, and also in the $H$-band, whereas it is not visible in the $J$-band images. This can be seen from Fig. 8 showing the $J$ and $H$-band images (which have lost a little in reproduction). The position of V445 Pup is circled. Due to its absence in the $J$-band, only a lower limit could be put for the $J$ magnitude by comparing the faintest, detected star in our $J$ image with its corresponding 2MASS $J$ magnitude. The limiting magnitude of the $J$-band image of 1 Nov. 2001 is 15.5. Our $J H K$ images/magnitudes confirm that the object was shrouded in thick dust shell by then. A similar conclusion was drawn earlier by Henden et al. (2001) based on observations of September-October 01. Henden et al. (2001) find that the star is not detectable in the $V$ and $I$-bands down to limiting magnitudes of 20 and 19.5 respectively - in the $K^{\prime}$-band the reported magnitude is 9.15 . The light curve of Fig. 1 also shows the steep decline in brightness, at around this time, indicating the onset of a heavy dust formation phase.

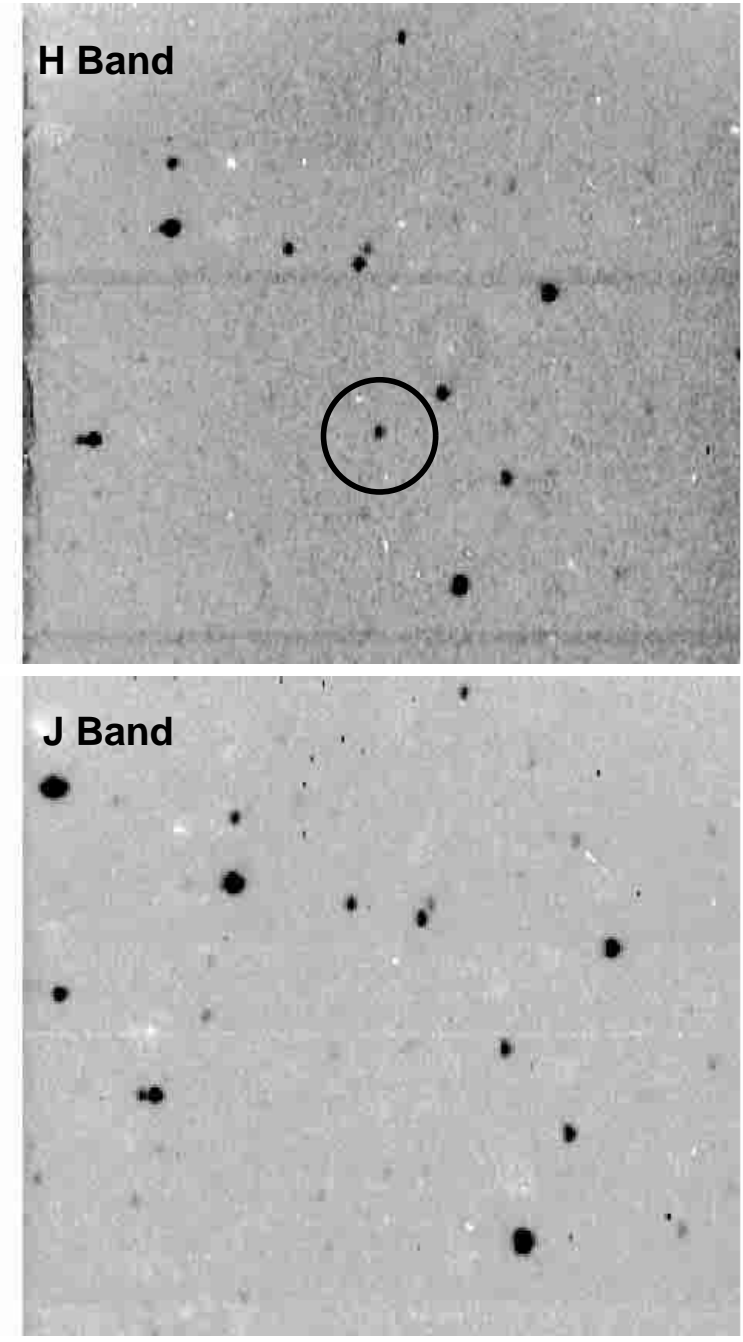

Fig. 8. $J$ and $H$-band images $(4 \times 4$ arcmin $)$ of V445 Puppis of 1 Nov. 2001. The object, which is circled in the $H$-band image, is not seen in the $J$-band. North is on the top and East is to the right.

\section{Discussion: The nature of V445 Puppis}

The nature of V445 Puppis is rather enigmatic. Among eruptive variables that show similar outbursts, one may first consider novae. V445 Puppis differs from a classical nova in the amplitude of its outburst and also the deficiency of hydrogen in its spectrum. The strong presence of a large number of carbon lines in the IR spectra is again unusual for a nova. Further, novae are known to evolve with time towards higher temperatures which characterize the nebular and coronal phases. Such behavior is not seen here. It also does not appear to be a recurrent nova because no earlier outbursts have been recorded or reported. In a symbiotic nova, one expects to see high-excitation emission lines in the spectrum during the decline phase - a feature which is absent here (Kenyon 1986). Also, the light curves of symbiotic novae decline much slower than what is observed in V445 Puppis. Most importantly, hydrogen deficiency is not expected in recurrent or symbiotic novae.

Another possibility is that V445 Puppis is a born-again AGB star. The nuclei of intermediate mass stars, evolving from the AGB stage into planetary nebulae can experience 
a final helium shell flash which lifts them into a high luminosity range again (Iben et al. 1983). There are only 3 known examples of born-again AGB's in the Galaxy viz. V605 Aql, FG Sge and Sakurai's object. However there are a few arguments against V445 Puppis being a born-again AGB. First, no nebulosity (caused by gas ionized by the hot nuclei) has been seen around the object as has been found in the other known cases. We have checked one of the $\mathrm{H} \alpha$ surveys covering this region (Schwartz 1990) but the object is not listed as showing signs of emission. Again, born-again AGB's - unlike V445 Puppis - brighten very gradually to their peak brightness - a process which can even take decades. For the actual timescales observed, the reader may refer to Duerbeck et al. (2000) for Sakurai's object, Harrison (1996) for V605 Aql and Fig. 1 of Blocker \& Schonberner (1997) for FG Sge.

There is a class of eruptive variables whose spectrum after the outburst evolves into that of a cool M-type giant or supergiant. This spectral evolution takes place rather rapidly - within a time of 1 to 2 months. The first such object in this class is a luminous, red variable star (M $31 \mathrm{RV}$ ) that erupted in M 31 (Rich et al. 1989; Mould et al. 1990). Subsequently the eruptive variable V4332 Sgr (Martini et al. 1999) and most recently V838 Mon (Banerjee \& Ashok 2002a; Munari et al. 2002) have been added to this class. A consensus has not been reached on placing these objects in the same category but what is certain is that after a nova-like outburst they quickly evolve to a very cool M type (or even later type) absorption spectrum. V838 Mon has, in fact, evolved at present to be similar to that of a $\mathrm{T}$ type brown dwarf (Banerjee \& Ashok 2002b; Geballe et al. 2002). In the case of V445 Puppis, there is VSNET photometric data in the $B V R I$-bands for more than 5 months after its outburst. We have checked these colors and do not find an evolutionary trend towards M-type giant/supergiant stage. For example on 2001, March 30.48 (more than 4 months after the outburst) the observed magnitudes are $B=10.66$ and $V=10.13$. For an adopted value of $E(B-V)=0.25$, this results into an intrinsic $(B-V)$ color of 0.29 which is very different from $\sim 1.6$ which is the expected value for a cool $\mathrm{M}$ giant/supergiant star. Thus V445 Puppis does not appear to show an evolutionary trend similar to the V838 Mon class of objects.

V445 Puppis also does not appear to be an RCB or hydrogen deficient carbon star (HdC). RCB stars show episodic dimming due to ejection of dusty carbon shells, subsequently followed by a rebrightening. But their spectra are typically $F$ type absorption spectra and most of them are detected in IRAS and show an IR excess. V445 Puppis was not detected previously by IRAS. Furthermore, no other outbursts have been recorded for V445 Puppis. HdC stars share most of the properties of RCB stars except that they do not show large brightness variations. Both groups of objects have absolute magnitudes $M_{V}$ in the range -3 to -5 (Brunner et al. 1998). Unless the extinction $A_{V}$ and distance $d$ to V445 Puppis are grossly underestimated, $M_{V} \sim 0$. This is inferred from $m_{V}-M_{V}=5 \log d-5+A_{V}$ using adopted values of $A_{V} \sim 1$, an upper limit for $d=3 \mathrm{kpc}$ and the pre-outburst magnitude value of $m_{V}=13.6$. The object appears under luminous for an RCB star.

The possibility that V445 Puppis is not a single star - but part of a binary system with an accretion disk - has some supporting evidence. First, the SED of the star in the preoutburst stage (Fig. 7, lower panel) is difficult to fit with a black-body spectrum. We have tried several black-body fits at different temperatures to the observed SED. While fitting, the observed SED has itself been modified by choosing different $E(B-V)$ values in case the adopted value of $E(B-V)=0.25$ is grossly incorrect. However a good fit is notfound and the closest fit to the data $(T=6500 \mathrm{~K}, E(B-V)=0.25)$ is not satisfactory as can be seen from Fig. 7 (lower panel). It is possible that the observed continuum distribution from V445 Puppis is dominated by radiation from an accretion disk surrounding the object. In the case of a steady-state accretion disk around a white-dwarf, the continuum radiation from the disk can be described by an $F_{v} \alpha v^{1 / 3}$ relation (Mayo et al. 1980 and references therein). A $F_{v} \alpha v^{1 / 3}$ dependence falls off less steeply than the $F_{v} \alpha v^{2}$ relation expected for a black-body in the RayleighJeans regime. We find that such an accretion disk spectrum fits the data of Fig. 7 much better than a black-body fit.

The radio data from Rupen et al. (2001a,b and their website http://www. aoc.nrao.edu/mrupen/XRT/V445Pup) also suggest the possibility of an accretion process in a binary system. They state that theobserved synchrotron emission probably originates either in accretion onto a compact companion or in an asymmetric, clumpy shock at the boundary between ejecta from a fresh outburst in September 2001 and previous ejecta (the fresh outburst would be obscured by the thick dust shell which had formed around this time). But they argue that the rapid evolution of both the radio emission and the radio absorption - significant flaring is seen in the object - favors the presence of an accretion process. However, it must be pointed out, there is a lacuna in comparing the observed radio emission with radiation from an accretion disk with a $v^{1 / 3}$ spectra as discussed above. The latter does not constitute a non-thermal source like the observed radio emission. But the present radio detection was made well after the outburst. It may just be possible that the physical conditions/mechanisms of the accretion process are different in the pre- and post-outburst stages leading to a qualitative difference in the radiation emitted. We are unable to judge on this aspect. The primary aim of the present work was to look for any evidence - however slight - which would show whether V445 Puppis belongs to a binary system or not. Given the strangeness of the object, this would help understand its nature. We feel that the recent radio data and the pre-outburst SED seem to indicate a binary nature for V445 Puppis.

From the arguments given above, we find that it is difficult to place V445 Puppis into known categories of eruptive variables. Kato et al. (1989) and Iben \& Tutukov (1994), among other workers, have investigated the case of Helium novae. Kato et al. (1989) consider the case of a degenerate white dwarf accreting helium from its helium-rich companion. For appropriate mass accretion rates, an accretion-induced collapse of the white dwarf into a neutron star need not occur. Instead a thermo-nuclear reaction can occur on the surface of the white dwarf leading to a Helium nova outburst. The ejecta of such an outburst, in the Kato et al. (1989) model, should presumably be rich in helium and carbon and highly hydrogen-deficient. This is based on the assumed, pre-ignition, chemical 
composition of the envelope by mass, viz $X=0, Y=0.97$ and $Z=0.03$. Further, carbon in the ejecta is expected to be additionally enhanced because the thermonuclear outburst process itself converts helium to carbon. Such a scenario, or some close variant of it, may apply to V445 Puppis and explain the hydrogen deficiency (and $\mathrm{He} / \mathrm{C}$ enrichment) of its optical and IR spectra. The tentative evidence for binarity and accretion disk in V445 Puppis is also in conformity with the requirements of a Helium nova scenario. However, the predicted luminosity of the outburst in a Helium nova is $L \sim 10^{5} L_{\odot}$ (Kato et al. 1989) and X-rays are also predicted in the post-outburst stage (Iben \& Tutukov 1994). In the case of V445 Puppis, there are no reports on X-ray detection from the object, though it is uncertain whether any attempt has been made in this direction. Further the thick dust shell may attenuate any soft X-ray emission that takes place. The outburst luminosity, if approximated from the SED of 2 Jan. 01 (Fig. 7), is found to be closer to $10^{4} L_{\odot}$ i.e. slightly less than the theoretical estimate. In view of this, it may be slightly speculative to identify V445 Puppis as a Helium nova, but similarities exist. Given the interesting and unique properties of the object, it is hoped that this work will prompt a rigorous, theoretical study to explain the nature of V445 Puppis.

Acknowledgements. The research work at Physical Research Laboratory is funded by the Department of Space, Government of India. We thank A.Tej for help in obtaining observations. We thank R. M. Wagner, the referee, for useful and constructive comments that have helped improve the paper. This work has made use of data available from 2MASS data center and also from data/information available at the following websites viz. http://kurucz.harvard.edu/linelists.html; http://vela.as.arizona. edu/ rmw/v445pup.html; http://www. kusastro.kyoto-u.ac.jp.vsnet, http://www. aoc.nrao. edu/ mrupen/XRT/V445Pup and http://www1.harenet.ne.jp/ aikow/0113pnp.gif

\section{References}

Allen, C. W. 1976, Astrophysical Quantities (London and Dover: Athlone Press)

Aller, L. H. 1963, The Atmospheres of the Sun and Stars (New York: Ronald Press)

Ashok, N. M., \& Banerjee, D. P. K. 2001, IAU Circ., 7559

Banerjee, D. P. K., \& Ashok, N. M. 2002a, A\&A, 395, 161

Banerjee, D. P. K., \& Ashok, N. M. 2002b, IAU Circ., 8036

Blocker, T., \& Schonberner, D. 1997, A\&A, 324, 991

Brunner, A. R., Clayton, G. C., \& Ayres, T. R. 1998, PASP, 110, 1412

Chandrasekhar, T., Ashok, N. M., \& Ragland, S. 1992, MNRAS, 255, 412

Duerbeck, H. W., Liller, W., Sterken, C., et al. 2000, AJ, 119, 2360
Eenens, P. R. J., Williams, P. M., \& Wade, R. 1991, MNRAS, 252, 300

Fujii, M. 2001, IAU Circ., 7556

Geballe, T. R., Smalley, B., Evans, A., \& Rushton, M. T. 2002, IAU Circ., 8016

Gehrz, R. D. 1988, ARA\&A, 26, 377

Gilmore, A. C. 2001, IAU Circ., 7559

Harrison, T. E. 1996, PASP, 108, 1112

Henden, A. A., Wagner, R. M., \& Starrfield, S. G. 2001, IAU Circ., 7730

Hunt, L. K., Manucci, F., Testi, L., et al. 1998, AJ, 115, 2594

Iben, I. Jr., Kaler, J. B., Truran, J. W., \& Renzini, A. 1983, ApJ, 264, 605

Iben, I. Jr., \& Tutukov, A. V. 1994, ApJ, 431, 264

Johansson, L., \& Litzen, U. 1965, Ark. for Fysik Band 29, 13, 165

Johansson, L. 1966, Ark. for Fysik Band 31, 15, 201

Kamath, U. S., \& Anupama, G. S. 1992, BASI, 30, 679

Kenyon, S. J. 1976, The Symbiotic Stars (Cambridge, UK: Cambridge Univ. Press)

Koornneef, J. 1983, A\&A, 128, 84

Kato, M., Saio, H., \& Hachisu, I. 1989, ApJ, 340, 509

Kato, T., \& Kanatsu, K. 2002, IAU Circ., 7552

Lang, K. R. 1991, Astrophysical data: Planets and Stars (New York: Springer-Verlag)

Liller, W. 2001a, IAU Circ., 7556

Liller, W. 2001b, IAU Circ., 7561

Lynch, D. K., Russel, R. W., \& Sitko, M. L. 2001, AJ, 122, 3313

Martini, P., Wagner, R., Tomaney, A., et al. 1999, AJ, 118, 1034

Mayo, S. K., Wickramasinghe, D. T., \& Whelan, J. A. J. 1980, MNRAS, 193793

Mould, J., Cohen, J., Graham, J. R., et al. 1990, ApJ, 353, L35

Munari, U., Henden, A., Kiyota, S., et al. 2002a, A\&A, 389, L51

Neckel, Th., Klare, G., \& Sarcander, M. 1980, A\&AS, 42, 251

Platais, I., Kozhurina-Platais, V., \& Zacharias, M. I., et al. 2002, IAU Circ., 7556

Rich, R. M., Mould, J., Picard, A., Frogel, J. A., \& Davies, R. 1989, ApJ, 341, L51

Rupen, M. P., Dhawan, V., \& Mioduszewski, A. 2001a, IAU Circ., 7717

Rupen, M. P., Dhawan, V., \& Mioduszewski, A. 2001b, IAU Circ., 7728

Schwartz, R. D., Persson, S. E., \& Hamann, F. W. 1990, AJ, 100, 793

Seaquist, E. R. 1989, in Classical Novae, ed. M. F. Bode, \& A. Evans (Oxford: John Wiley)

Shemmer, O., Leibowitz, E., \& Dan, J. 2001, IAU Circ., 7556

Wagner, R. M., Foltz, C. B., \& Starrfield, S. G. 2001a, IAU Circ., 7556

Wagner, R. M., Schwarz, G., \& Starrfield, S. G. 2001b, IAU Circ., 7571

Wagner, R. M., Schwarz, G., \& Starrfield, S. G. 2001c, IAU Circ., 7717

Williams, R. E. 1994, ApJ, 426, 279

Wooden, W. H. II. 1970, AJ, 75, 324

Woodward, C. E., Lawrence, G. F., Gehrz, R. D., et al. 1993, ApJ, 408, L37 